\title{
The Bose-Hubbard ground state: extended Bogoliubov and variational methods compared with time-evolving block decimation
}

\author{
Ippei Danshita ${ }^{1}$ and Pascal Naidon ${ }^{2}$ ' \\ ${ }^{1}$ Department of Physics, Faculty of Science, Tokyo University of Science, Shinjuku-ku, Tokyo 162-8601, Japan \\ ${ }^{2}$ ERATO Macroscopic Quantum Project, JST, Tokyo, 113-0033 Japan
}

\begin{abstract}
We determine the ground-state properties of a gas of interacting bosonic atoms in a one-dimensional optical lattice. The system is modelled by the Bose-Hubbard Hamiltonian. We show how to apply the time-evolving block decimation method to systems with periodic boundary conditions, and employ it as a reference to find the ground state of the Bose-Hubbard model. Results are compared with recently proposed approximate methods, such as Hartree-Fock-Bogoliubov (HFB) theories generalised for strong interactions and the variational BijlDingle-Jastrow method. We find that all HFB methods do not bring any improvement to the Bogoliubov theory and therefore provide correct results only in the weakly-interacting limit, where the system is deeply in the superfluid regime. On the other hand, the variational Bijl-Dingle-Jastrow method is applicable for much stronger interactions, but is essentially limited to the superfluid regime as it reproduces the superfluid-Mott insulator transition only qualitatively.
\end{abstract}

PACS number: 03.75.Hh, 03.75.Lm, 05.30.Jp

Keywords: optical lattice, superfluid-to-Mott insulator transition, Hartree-Fock-Bogoliubov, Bijl-Dingle-Jastrow, TEBD

\section{INTRODUCTION}

Optical lattices loaded with cold atomic gases have provided a fertile experimental testing ground for the study of fundamental phenomena exhibited by quantum degenerate gases [1, 2, 3]. One of the greatest advantages of these systems is that microscopic theories can be directly compared with experiments without the use of any fitting parameters thanks to the diluteness of atomic gases and the flexible and precise controllability of parameters. In particular, the Bose-Hubbard model [4, 5] has been successful in treating systems of cold bosonic atoms confined in optical lattices and explaining their various intriguing physical properties observed in experiments, such as the superfluid-Mott insulator transition [6, 7, 8], the superfluid critical velocities [9, 10], and the quantum depletion of condensates [11, 12].

The one-dimensional (1D) Bose-Hubbard model has the following form

$$
\hat{H}_{\mathrm{bh}}=-J \sum_{l=1}^{M}\left(\hat{a}_{l}^{\dagger} \hat{a}_{l+1}+\text { h.c. }\right)+\frac{U}{2} \sum_{l=1}^{M} \hat{n}_{l}\left(\hat{n}_{l}-1\right)
$$

where $\hat{a}_{l}\left(\hat{a}_{l}^{\dagger}\right)$ creates a boson at the lowest level localised on the $l$-th site of a 1D lattice, and $\hat{n}_{l}=\hat{a}_{l}^{\dagger} \hat{a}_{l}$ is the number operator. $J$ is the hopping energy from one site to the nearest neighbor, and $U>0$ is the onsite repulsion. When the total number of particles $N$ is incommensurate with the number of sites $M$, the system is in a superfluid state at zero temperature. When $N$ is commensurate with $M$, the system is superfluid for small $U / J$ and becomes a Mott insulator for large $U / J$.

In the superfluid phase, several approximations are available to treat this Hamiltonian analytically. The most famous one is the Bogoliubov approximation [13]. However, it is limited to

\footnotetext{
*Electronic address: pascal@cat.phys.s.u-tokyo.ac.jp
}

very weak interactions, i.e. $U /(n J) \ll 1$, where $n=N / M$ is the filling factor. It would be desirable to extend this approximation to stronger interactions. In the context of magneticallytrapped ultracold atomic gases, several extensions of the Bogoliubov approximation have been proposed in order to accurately reproduce the condensate fraction and the collective mode frequencies observed in experiments. These extensions are based on the Hartree-Fock-Bogoliubov (HFB) approximation [14], which is closely related to the Girardeau-Arnowitt pair theory [38]. The most used one is the HFB-Shohno approximation [16] (also known as HFB-Popov [17]). However, in this approximation, the condensate-condensate correlation is removed by hand to ensure a gapless excitation spectrum. Several authors have suggested an improved HFB approximation by introducing that correlation by hand so that a gapless excitation spectrum is preserved [18, 19]. More recently, Yukalov et al. [20, 21] proposed a treatment of the HFB approximation in the context of representative statistical ensembles which preserves both the correlation and absence of a gap, and aims at describing the strongly-interacting regime.

Another line of approach at zero temperature is the variational method, which consists in minimizing the energy within a given subspace of the Hilbert space. The accuracy and complexity of the method depend on the choice of the subspace. Recently, the Bijl-Dingle-Jastrow form [22, 23] was proposed as an interesting variational ansatz for bosons in a lattice [24].

The purpose of this paper is to examine the "performance" of these theories in the case of a 1D lattice system. An advantage of lattice systems is that they eliminate issues and ambiguities associated with ultraviolet divergences found in continuous systems with contact interactions. We choose a 1D system because low dimensionality increases the effects of quantum fluctuations and therefore the possible differences between the theories. Moreover, for 1D systems, we can obtain quasi-exact numerical solutions employing the time-evolving block decimation (TEBD) method [25], which can be used as a reference. To put some perspective about the computational effort required by these methods, we should note that Bogoliubovrelated calculations typically take several seconds, variational Bijl-Dingle-Jastrow calculations several minutes, and TEBD calculations several days[41].

The paper is organised as follows: we first explain the TEBD method in Section [I] Especially, the explanation is focused on 
the application of the TEBD method to systems with a periodic boundary condition. The extended Bogoliubov methods (Bogoliubov, HFB, HFB-Shohno, improved-HFB, and HFBYukalov) are detailed in Section III. The Bijl-Dingle-Jastrow method is presented in Section IV Finally, we compare all these methods numerically in Section $V$.

\section{THE TEBD METHOD}

Since the main purpose of this work is to examine the performance of several approximate approaches, accurate groundstates obtained by a quasi-exact numerical method are necessary for a reference. The TEBD method is a variant of the density matrix renormalization group (DMRG) method [26, 27], which is one of the best methods available to study 1D quantum lattice systems, and provides accurate time evolutions of many-body wave functions and ground states via an imaginary time evolution. Although homogeneous systems with periodic boundary conditions are better suited for the analytical approaches that we will use later, so far the TEBD method has been applied to systems with open [25] or infinite [28] boundary conditions. In this section, we present a detailed explanation on how to apply the TEBD algorithm to systems with periodic boundary conditions.

We consider a quantum lattice system with a periodic boundary condition composed of $M$ sites, which are labeled by index $l, l \in\{1, \ldots, M\}$. We assume that the Hamiltonian consists of only on-site and nearest-neighbor terms and is written as

$$
\hat{H}=\sum_{l=1}^{M}\left(\hat{K}_{1}^{[l]}+\hat{K}_{2}^{[l, l+1]}\right)
$$

where $\hat{K}_{2}^{[M, M+1]} \equiv \hat{K}_{2}^{[M, 1]}$, reflecting the periodic boundary condition. In the case of the Bose-Hubbard model, for example, $\hat{K}_{1}^{[l]}$ and $\hat{K}_{2}^{[l, l+1]}$ correspond to the on-site interaction and the hopping. Spanning the Hilbert space of the whole system by a product of local Hilbert spaces of dimension $d$, a many-body wave function of the system can be expressed as

$$
|\Psi\rangle=\sum_{j_{1}, j_{2}, \ldots, j_{M}=1}^{d} c_{j_{1}, j_{2}, \ldots, j_{M}}\left|j_{1}\right\rangle\left|j_{2}\right\rangle \cdots\left|j_{M}\right\rangle
$$

In the TEBD algorithm, coefficients $c_{j_{1}, j_{2}, \ldots, j_{M}}$ are decomposed in a particular matrix product form as

$$
\begin{aligned}
c_{j_{1}, j_{2}, \ldots, j_{M}}= & \sum_{\alpha_{1}, \ldots, \alpha_{M-1}=1}^{\chi} \Gamma_{\alpha_{1}}^{[1] j_{1}} \lambda_{\alpha_{1}}^{[1]} \Gamma_{\alpha_{1} \alpha_{2}}^{[2] j_{2}} \lambda_{\alpha_{2}}^{[2]} \ldots \lambda_{\alpha_{M-2}}^{[M-2]} \\
& \times \Gamma_{\alpha_{M-2} \alpha_{M-1}}^{[M-1] j_{M-1}} \lambda_{\alpha_{M-1}}^{[M-1]} \Gamma_{\alpha_{M-1}}^{[M] j_{M}}
\end{aligned}
$$

The vector $\lambda_{\alpha_{l}}^{[l]}$ represents the coefficients of the Schmidt decomposition of $|\Psi\rangle$ with respect to the bipartite splitting of the system into $[1, \ldots, l-1, l]:[l+1, l+2, \ldots, M]$,

$$
|\Psi\rangle=\sum_{\alpha_{l}=1}^{\chi} \lambda_{\alpha_{l}}^{[l]}\left|\Phi_{\alpha_{l}}^{[1, \ldots, l-1, l]}\right\rangle\left|\Phi_{\alpha_{l}}^{[l+1, l+2, \ldots, M]}\right\rangle
$$

where the Schmidt vectors $\left|\Phi_{\alpha_{l}}^{[1, \ldots, l-1, l]}\right\rangle$ and $\left|\Phi_{\alpha_{l}}^{[l+1, l+2, \ldots, M]}\right\rangle$ are expressed as

$$
\begin{aligned}
\left|\Phi_{\alpha_{l}}^{[1, \ldots, l-1, l]}\right\rangle & =\sum_{j_{1}, \ldots, j_{l}=1}^{d} \sum_{\alpha_{1}, \ldots, \alpha_{l-1}=1}^{\chi} \Gamma_{\alpha_{1}}^{[1] j_{1}} \lambda_{\alpha_{1}}^{[1]} \cdots \lambda_{\alpha_{l-2}}^{[l-2]} \\
& \left.\times \Gamma_{\alpha_{l-2} \alpha_{l-1}}^{[l-1] j_{l-1}} \lambda_{\alpha_{l-1}}^{[l-1]} \Gamma_{\alpha_{l-1} \alpha_{l}}^{[l] j_{l}} \alpha_{1}\right\rangle \cdots\left|j_{l-1}\right\rangle\left|j_{l}\right\rangle
\end{aligned}
$$

and

$$
\begin{aligned}
\left|\Phi_{\alpha_{l}}^{[l+1, l+2, \ldots, M]}\right\rangle= & \sum_{j_{l+1}, \ldots, j_{M}=1}^{d} \sum_{\alpha_{l+1}, \ldots, \alpha_{M}=1}^{\chi} \Gamma_{\alpha_{l} \alpha_{l+1}}^{[l+1] j_{l+1}} \lambda_{\alpha_{l+1}}^{[l+1]} \\
& \times \Gamma_{\alpha_{l+1} \alpha_{l+2}}^{[l+2] j_{l+2}} \lambda_{\alpha_{l+2}[l+2]}^{[l+2} \ldots \lambda_{\alpha_{M-1}}^{[M-1]} \Gamma_{\alpha_{M-1}}^{[M] j_{M}} \\
& \times\left|j_{l+1}\right\rangle\left|j_{l+2}\right\rangle \cdots\left|j_{M}\right\rangle,
\end{aligned}
$$

respectively. In general, the number of basis configurations $\chi$ required for convergence is of the order $d^{L / 2}$ in order to express arbitrary states [25]. However, since the Schmidt coefficients $\lambda_{\alpha_{l}}^{[l]}$ decay rapidly as the index $\alpha_{l}$ increases for the ground state or low-lying excited states, the TEBD method with relatively small number of states $\chi$ can be quasi-exact for these states.

In order to compute the time evolution of a state, i.e. $e^{-i \hat{H} t}|\Psi\rangle$, we split the Hamiltonian into three parts $\hat{H}_{\text {odd }}=$ $\sum_{1 \leq m \leq M / 2}\left(\hat{K}_{1}^{[2 m-1]}+\hat{K}_{2}^{[2 m-1,2 m]}\right), \hat{H}_{\text {even }}=\sum_{1 \leq m<M / 2}\left(\hat{K}_{1}^{[2 m]}+\right.$ $\left.\hat{K}_{2}^{[2 m, 2 m+1]}\right)$, and $\hat{H}_{\text {edge }}=\hat{K}_{1}^{[M]}+\hat{K}_{2}^{[M, 1]}$. Subsequently, we use the second-order Suzuki-Trotter expansion to decompose $e^{-i \hat{H} t}$ into a product of two-site operators. When the number of lattice sites $M$ is even,

$$
\begin{aligned}
e^{-i \hat{H} \delta}= & e^{-\frac{i \hat{H}_{\mathrm{odd}} \delta}{2}} e^{-i \hat{H}_{\mathrm{even}} \delta} e^{-i \hat{H}_{\mathrm{edge}} \delta} e^{-\frac{i \hat{H}_{\mathrm{odd}} \delta}{2}} \\
& +O\left(\delta^{3}\right)
\end{aligned}
$$

because $\left[\hat{H}_{\text {even }}, \hat{H}_{\text {edge }}\right]=0$. On the other hand,

$$
\begin{aligned}
e^{-i H \delta}= & e^{-\frac{i \hat{H}_{\mathrm{odd}} \delta}{2}} e^{-\frac{i \hat{H}_{\mathrm{even}} \delta}{2}} e^{-i \hat{H}_{\mathrm{edge}} \delta} e^{-\frac{i \hat{H}_{\mathrm{even}} \delta}{2}} e^{-\frac{i \hat{H}_{\mathrm{odd}} \delta}{2}} \\
& +O\left(\delta^{3}\right)
\end{aligned}
$$

for odd $M$. The operators $e^{-\frac{i \hat{H}_{\text {odd }} \delta}{2}}$ and $e^{-\frac{i \hat{H}_{\mathrm{even}} \delta}{2}}$ can be efficiently applied because they act on two neighboring $\Gamma$ tensors [25]. However, in order to apply $e^{-i \hat{H}_{\text {edge }} \delta}$, one needs to deform the tensor product Eq. 4 to place $\Gamma^{[M]}$ next to $\Gamma^{[1]}$; this deformation is achievable by means of the swapping techniques [30].

The swapping techniques allow us to exchange the positions of two neighboring $\Gamma$ tensors in the tensor product and have been successfully applied to extend the TEBD to the two-legged ladder geometry [31] or two-component Bose gases [32]. We briefly review the swapping operation introduced in Ref. [30], because it is crucial for the application of the TEBD to systems with periodic boundary conditions. For simplicity, we shall explain how to swap the positions of $\Gamma^{[l]}$ and $\Gamma^{[l+1]}$. Let us express the state in a local basis that focuses on sites $l$ and $l+1$ as

$$
\begin{aligned}
|\Psi\rangle= & \sum_{j_{l}, j_{l+1}=1 \alpha_{l-1}, \alpha_{l+1}=1}^{d} \sum_{\alpha_{l-1} \alpha_{l+1}}^{\chi} j_{j} j_{l+1} \\
& \times\left|\Phi_{\alpha_{l-1}}^{[1, \ldots, l-1]}\right\rangle\left|j_{l}\right\rangle\left|j_{l+1}\right\rangle\left|\Phi_{\alpha_{l+1}}^{[l+2, \ldots, M]}\right\rangle,
\end{aligned}
$$



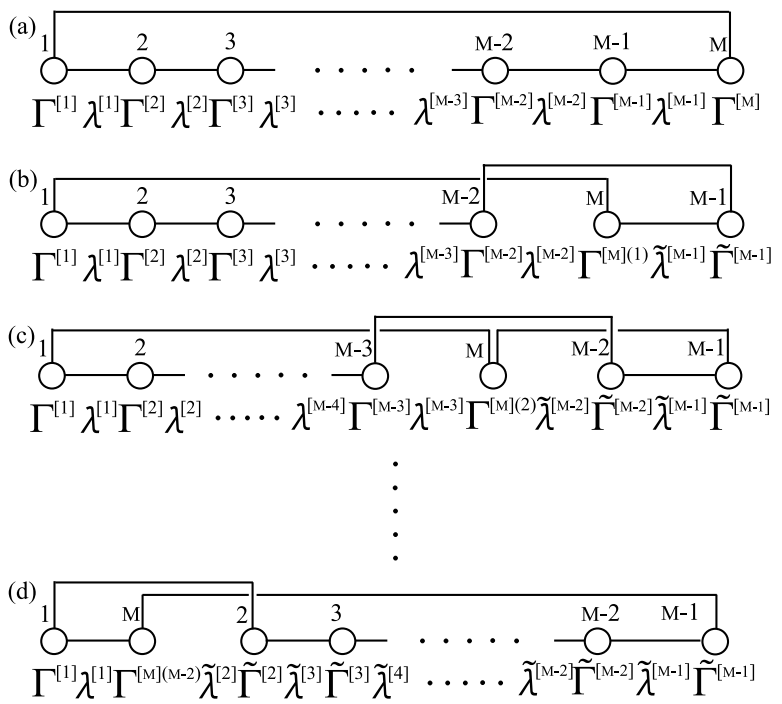

Figure 1: Schematic picture explaining how to apply $e^{-i \hat{H}_{\text {edge }} \delta}$ to a state by using the swapping techniques. Open circles represent lattice sites and solid lines correspond to the connections of the lattice sites via the nearest-neighbor interactions. (a) expresses a tensor product in the form of Eq. (4), In (b), $\Gamma^{[M-1]}$ and $\Gamma^{M]}$ are swapped and the new tensors are expressed as $\Gamma^{[M](1)}$ and $\tilde{\Gamma}^{[M-1]}$. In (c), subsequently, $\Gamma^{[M-2]}$ and $\Gamma^{[M](1)}$ are swapped and the new tensors are expressed as $\Gamma^{[M](2)}$ and $\tilde{\Gamma}^{[M-2]}$. (d) expresses a tensor product after conducting the swapping $M-2$ times. Since $\Gamma^{[1]}$ and $\Gamma^{[M](M-2)}$ are neighboring in (d), one can efficiently apply $e^{-i \hat{H}_{\text {edge }} \delta}$.

where

$$
\Theta_{\alpha_{l-1} \alpha_{l+1}}^{j_{l} j_{l+1}}=\sum_{\alpha_{l}=1}^{\chi} \lambda_{\alpha_{l-1}}^{[l-1]} \Gamma_{\alpha_{l-1} \alpha_{l}}^{[l] j_{l}} \lambda_{\alpha_{l}}^{[l]} \Gamma_{\alpha_{l} \alpha_{l+1}}^{[l+1] j_{l+1}} \lambda_{\alpha_{l+1}}^{[l+1]}
$$

In order to decompose $\Theta_{\alpha_{l-1} \alpha_{l+1}}^{j_{l} j_{l+1}}$ back into the tensor product form as the right-hand side of Eq. [11), one has to reshape the fourth-order tensor $\Theta_{\alpha_{l-1} \alpha_{l+1}}^{j_{l} j_{l+1}}$ into a matrix $\Theta_{\left\{j_{l}, \alpha_{l-1}\right\},\left\{j_{l+1}, \alpha_{l+1}\right\}}$ of the dimension $d \chi \times d \chi$, where $\alpha_{l-1}$ and $\alpha_{l+1}$ are coupled with $j_{l}$ and $j_{l+1}$ respectively, and conduct the singular value decomposition of the matrix. In the swapping process, by coupling $\alpha_{l-1}$ with $j_{l+1}$ and $\alpha_{l+1}$ with $j_{l}$, one reshapes the $\Theta$ tensor as $\Theta_{\alpha_{l-1} \alpha_{l+1}}^{j_{l} j_{l+1}} \rightarrow \tilde{\Theta}_{\left\{j_{l+1}, \alpha_{l-1}\right\},\left\{j_{l}, \alpha_{l+1}\right\}}$. The singular value decomposition of the matrix $\tilde{\Theta}_{\left\{j_{l+1}, \alpha_{l-1}\right\},\left\{j_{l}, \alpha_{l+1}\right\}}$ results in the swapped form of the tensor product,

$$
\Theta_{\alpha_{l-1} \alpha_{l+1}}^{j_{l} j_{l+1}}=\sum_{\alpha_{l}=1}^{\chi} \lambda_{\alpha_{l-1}}^{[l-1]} \tilde{\Gamma}_{\alpha_{l-1}}^{l l+1] \tilde{\alpha}_{l}} \tilde{\lambda}_{\tilde{\alpha}_{l}}^{[l]} \tilde{\Gamma}_{\tilde{\alpha}_{l} \alpha_{l+1}}^{[l] j_{l}} \lambda_{\alpha_{l+1}}^{[l+1]} .
$$

The newly obtained vector $\tilde{\lambda}_{\tilde{\alpha}_{l}}^{[l]}$ corresponds to the coefficients of the Schmidt decomposition of $|\Psi\rangle$ with respect to the bipartition of the system into $[1, \ldots, l-1, l+1]:[l, l+2, \ldots, M]$,

$$
|\Psi\rangle=\sum_{\tilde{\alpha}_{l}=1}^{\chi} \tilde{\lambda}_{\tilde{\alpha}_{l}}^{[l]}\left|\tilde{\Phi}_{\tilde{\alpha}_{l}}^{[1, \ldots, l-1, l+1]}\right\rangle\left|\tilde{\Phi}_{\tilde{\alpha}_{l}}^{[l, l+2, \ldots, M]}\right\rangle .
$$

where

$$
\begin{aligned}
\left|\tilde{\Phi}_{\tilde{\alpha}_{l}}^{[1, \ldots, l-1, l+1]}\right\rangle= & \sum_{j_{l+1}=1}^{d} \sum_{\alpha_{l-1}=1}^{\chi} \lambda_{\alpha_{l-1}}^{[l-1]} \tilde{\Gamma}_{\alpha_{l-1}}^{[l+1] \tilde{\alpha}_{l}} \tilde{j}_{l+1} \\
& \times\left|\Phi_{\alpha_{l-1}}^{[1, \ldots, l-1]}\right\rangle\left|j_{l+1}\right\rangle
\end{aligned}
$$

$$
\begin{aligned}
\left|\tilde{\Phi}_{\tilde{\alpha}_{l}}^{[l, l+2, \ldots, M]}\right\rangle= & \sum_{j_{l}=1}^{d} \sum_{\alpha_{l+1}=1}^{\chi} \tilde{\Gamma}_{\tilde{\alpha}_{l} \alpha_{l+1}}^{[l] j_{l}} \lambda_{\alpha_{l+1}}^{[l+1]} \\
& \times\left|j_{l}\right\rangle\left|\Phi_{\alpha_{l+1}}^{[l+2, \cdots, M]}\right\rangle .
\end{aligned}
$$

Once the swapping techniques are introduced, it is straightforward to apply $e^{-i \hat{H}_{\text {edge }} \delta}$ to a state as indicated in Fig. 1 1 We first exchange positions of sites $M-1$ and $M$ (Fig. 1.b)) and next exchange positions of sites $M-2$ and $M$ (Fig. 11.c)). We continue conducting the swapping until the site $M$ becomes next to the first site as shown in Fig. 1(d). Then, $e^{-i \hat{H}_{\text {edge }} \delta}$ can be efficiently applied. Thus, the TEBD algorithm can be applied to systems with periodic boundary conditions by means of the swapping techniques.

Using the "periodic-TEBD" method explained above, we calculate the ground state of the Bose-Hubbard Hamiltonian (1) via imaginary time evolution

$$
\left|\Psi_{g}\right\rangle=\lim _{\tau \rightarrow \infty} \frac{e^{-\hat{H}_{\mathrm{bh}} \tau}\left|\Psi_{0}\right\rangle}{\| e^{-\hat{H}_{\mathrm{bh}} \tau}\left|\Psi_{0}\right\rangle}
$$

where $\left|\Psi_{0}\right\rangle$ is an initial state. We also use the numberconserving version of the TEBD method, which allows substantial speed-up of the simulations [33]. In the TEBD procedure, we choose the maximum number of atoms per site $n_{\max }=7(d=8)$ and retain $\chi=150$ states in the adaptively selected Hilbert space.

Having obtained the ground state, we can calculate any observables in principle by taking the average of an operator $\hat{O}$ as $\langle\hat{O}\rangle \equiv\left\langle\Psi_{g}|\hat{O}| \Psi_{g}\right\rangle$.

\section{EXTENDED BOGOLIUBOV METHODS}

The archetypical theory describing the weakly interacting Bose gas near its ground state is obtained by the Bogoliubov method [13]. This method follows from the observation that the gas should be close to a purely condensed system. One can therefore decompose the field operator in the form $\hat{a}_{j}=\hat{c} z_{j}+\hat{\theta}_{j}$, where $z_{j}$ is the condensate mode, and treat the noncondensate projection $\hat{\theta}_{j}$ as a small correction. Often, it is convenient to treat $\hat{c}$ as a number $\sqrt{N_{0}}$, where $N_{0}$ is the number of condensed atoms, while maintaining $\left\langle\hat{\theta}_{j}\right\rangle=0$. Although this replacement is inexact for finite-sized systems with a fixed number of particles, it does not affect the results concerning basic properties. The operator $\hat{\theta}_{j}$ is then regarded as describing quantum fluctuations around the classical field $\sqrt{N_{0}} z_{j}$.

The Bogoliubov approximation corresponds to treating the fluctuations to second order in the Hamiltonian. It results in a Hamiltonian which is quadratic in $\hat{\theta}$, and can be diagonalised in the so-called quasiparticle basis. The system can then be described in terms of the noncondensate density matrix $\tilde{n}_{j k}=$ $\left\langle\hat{\theta}_{j}^{\dagger} \hat{\theta}_{k}\right\rangle$ and noncondensate anomalous average $\tilde{m}_{j k}=\left\langle\hat{\theta}_{j} \hat{\theta}_{k}\right\rangle$. In the ground state, for a uniform system, the condensate mode $z_{j}$ is uniform and $\tilde{n}_{j k}$ and $\tilde{m}_{j k}$ are translationally invariant, so 

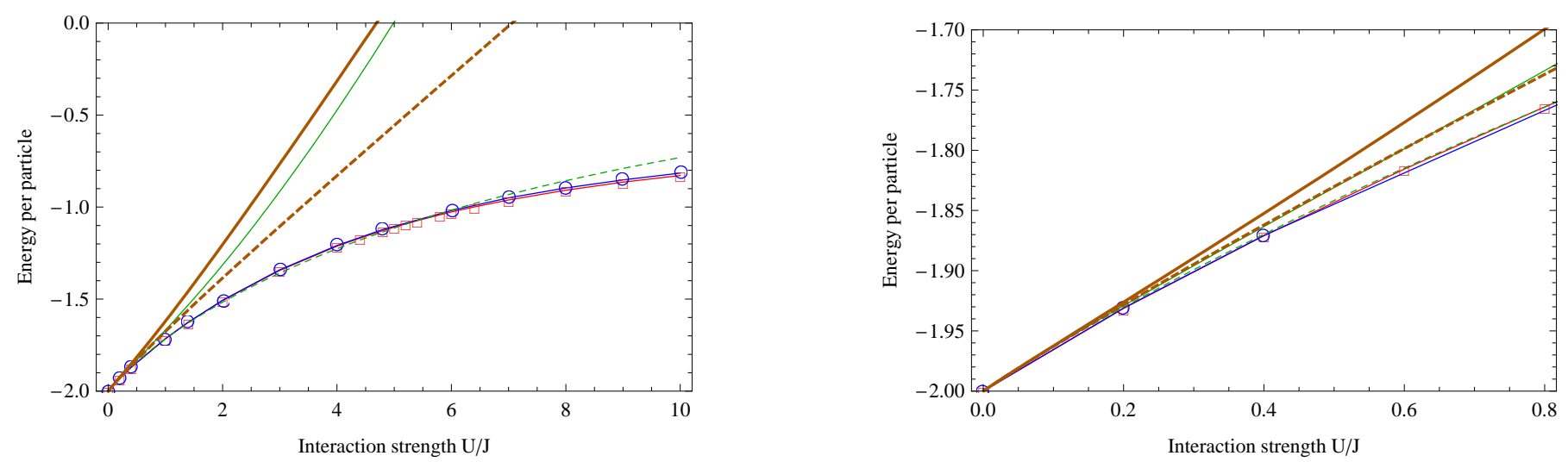

Figure 2: (Colour online) Energy per particle for $N=48, M=60$ as a function of $U / J$. The right panel is a close-up view of the weaklyinteracting regime. The red squares correspond to TEBD calculations, while the blue circles are obtained from the Jastrow variational method. The thin dashed green curve corresponds to the Bogoliubov result, the thin continuous green curve to the Popov result, while the thick dashed orange curve is the HFB result and the thick continuous orange curve is the improved HFB/Yukalov result.

that we can write:

$$
\begin{aligned}
z_{j} & =1 / \sqrt{M} \\
\tilde{n}_{j k} & =\frac{1}{M} \sum_{q=1}^{M-1} \tilde{n}_{q} e^{i \frac{2 \pi}{M} q \cdot(j-k)} \\
\tilde{m}_{j k} & =\frac{1}{M} \sum_{q=1}^{M-1} \tilde{m}_{q} e^{i \frac{2 \pi}{M} q \cdot(j-k)}
\end{aligned}
$$

where $\tilde{n}_{q}$ and $\tilde{m}_{q}$ are discrete Fourier transforms of $\tilde{n}_{j k}$ and $\tilde{m}_{j k}$, and $q$ is the lattice wave number. The $q=0$ mode (Goldstone mode) is omitted, which is consistent with the orthogonality between the condensate and noncondensate modes. We define the noncondensate and anomalous densities as:

$$
\begin{aligned}
& \tilde{n}=\frac{1}{M} \sum_{q=1}^{M-1} \tilde{n}_{q}, \\
& \tilde{m}=\frac{1}{M} \sum_{q=1} \tilde{m}_{q}
\end{aligned}
$$

and it follows from average number conservation that

$$
n_{0}=n-\tilde{n}
$$

where $n_{0}=N_{0} / M$ is the condensate density and $n=N / M$ is the total density.

Interactions generally tend to decrease the condensate fraction, and as a result the Bogoliubov approximation breaks down for sufficiently strong interactions. Especially it is unable to predict the Mott-insulator transition in commensurate lattice systems [34]. Even in incommensurate systems which remain superfluid, it is valid only in the weakly-interacting limit, which can be understood as follows. In the case of 1D Bose gases in continuum (with no lattice), the validity of the Bogoliubov approximation is judged by the dimensionless parameter $\gamma=l^{2} / \xi^{2}$ which expresses the competition between the aver-
The quasiparticle diagonalisation of the Hamiltonian leads to the following expressions for $\tilde{n}_{q}, \tilde{m}_{q}$ in the ground state:

$$
\begin{aligned}
& \tilde{n}_{q}=\frac{1}{2}\left(\frac{\omega_{q}}{\sqrt{\omega_{q}^{2}-\Delta^{2}}}-1\right), \\
& \tilde{m}_{q}=-\frac{1}{2} \frac{1}{\sqrt{\omega_{q}^{2}-\Delta^{2}}},
\end{aligned}
$$

with

$$
\begin{aligned}
\omega_{q} & =n U+4 J \sin \left(\frac{\pi q}{M}\right)^{2}, \\
\Delta & =n U,
\end{aligned}
$$

and the ground-state energy per site

$$
E=-2 J n+\frac{1}{2} U n^{2}+\frac{1}{2 M} \sum_{q=1}^{M-1}\left(\sqrt{\omega_{q}^{2}-\Delta^{2}}-\omega_{q}\right)
$$

One can also show that the excitation energies as a function of the excitation momentum are given by the quantity

$$
E_{q}=\sqrt{\omega_{q}^{2}-\Delta^{2}}
$$

age interparticle spacing $l$ and the healing length $\xi[35,36$. The healing length corresponds to the de Broglie wavelength associated with the 1D interaction energy. When $\gamma \ll 1$, the wave functions of the particles are well overlapped with each other and the gas can be approximately described by a macroscopic wave function (the condensate mode) with long-range phase coherence. Therefore, the Bogoliubov approximation is valid in this regime. As $\gamma$ increases, the overlap of the wave functions becomes smaller and the physical quantities, such as the ground state energy (24) and the excitation spectrum 25) 

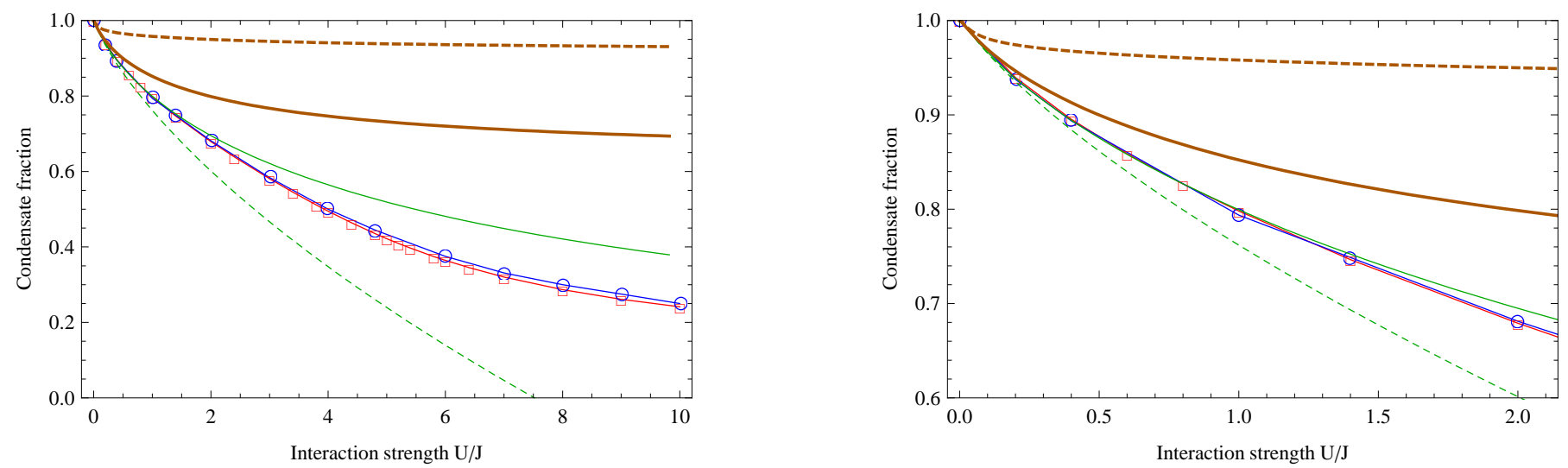

Figure 3: Condensate fraction for $N=48, M=60$ as a function of $U / J$. The right panel is a close-up view of the weakly-interacting regime. The same conventions as those of Fig. 2 are used.
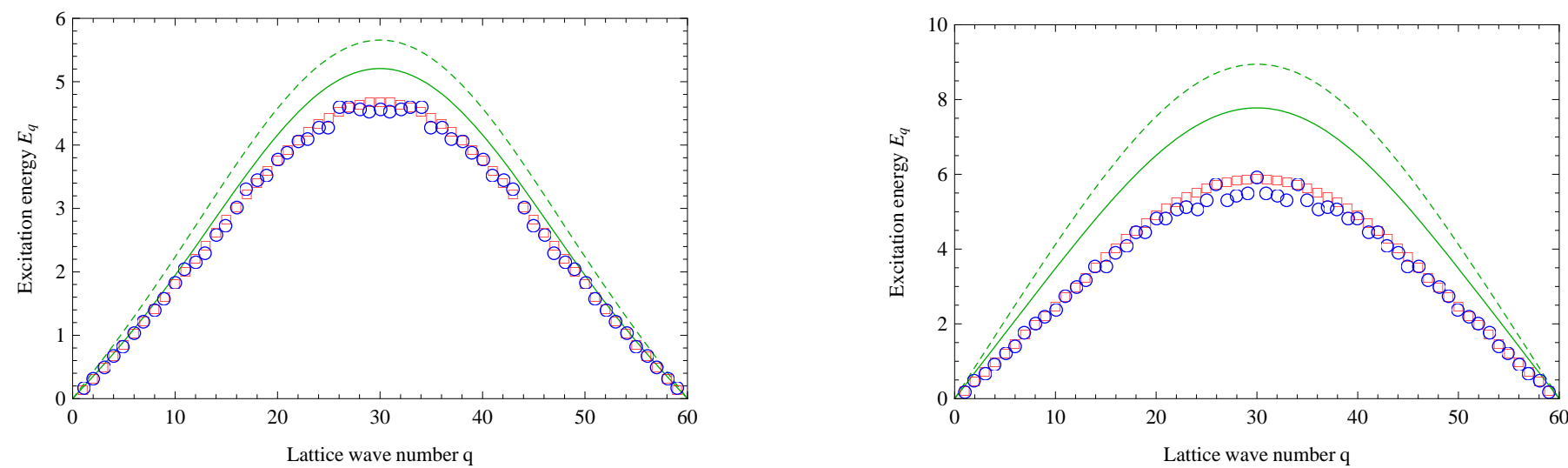

Figure 4: Excitation spectrum for $N=48, M=60$. The same conventions as those of Fig. 2 are used. The left panel corresponds to $U / J=2$ and the right panel corresponds to $U / J=8$. Note that the apparent dispersion of the blue circles are residual fluctuations due to the stochastic nature of the variational Monte-Carlo method.

deviate from those of the exact calculations based on the Bethe ansatz [37]. In the limit of $\gamma \gg 1$, long-range coherence disappears and the particles behave as impenetrable objects, which is referred to as the Tonks-Girardeau gas [38]. In the case of the Bose-Hubbard model, using $\xi=\hbar /\left(m^{*} c\right)[35]$ and $l=d / n$, the dimensionless parameter is expressed as $\gamma=U /(2 n J)$, where $m^{*}=\hbar^{2} /\left(2 J d^{2}\right)$ is the effective mass and $c=(2 n J U)^{1 / 2} d / \hbar$ is the Bogoliubov sound speed. Thus, the Bogoliubov approximation is valid only when $U \ll n J$.

One way to improve the Bogoliubov approximation is to include the higher-order perturbation terms, and make a decoupling approximation which reduces the Hamiltonian to a quadratic form again. This is the case when one assumes that the noncondensate particles are uncorrelated (using Wick's decoupling scheme for the operator $\hat{\theta}$ ). One then obtains the HFB approximation. The structure of the equations is the same as above, but now

$$
\begin{aligned}
\omega_{q} & =\left(n_{0}-\tilde{m}\right) U+4 J \sin \left(\frac{\pi q}{M}\right)^{2} \\
\Delta & =\left(n_{0}+\tilde{m}\right) U
\end{aligned}
$$

and the energy per site is

$$
E_{H F B}=E_{B o g}+U \tilde{n} \tilde{m}+\frac{1}{2} U\left(\tilde{n}^{2}-\tilde{m}^{2}\right)
$$

where $E_{B o g}$ refers to the expression on the right-hand side of Eq. 24) - note that it is not the Bogoliubov energy since $\omega_{q}$ and $\Delta$ have changed. The solution is now implicit, and Eqs. (17/18/19|20|21|26|27) have to be solved self-consistently.

While it retains the elegance of the Bogoliubov approximation, and is supposedly applicable to more strongly-interacting cases, the HFB approximation suffers from a notorious problem related to the existence of a gap in the excitation spectrum [17], which is forbidden in the superfluid regime according to the Hugenholtz-Pines theorem [39]. This gap can be expressed as $\lim _{q \rightarrow 0} E_{q}=2 U \sqrt{-\tilde{m} n_{0}}$, and clearly comes from the anomalous average $\tilde{m}$ which is supposed to be strictly negative, according to Eq. 21). One ad hoc solution to this problem, first used by Shohno [16], is to set $\tilde{m}$ to zero by hand in the HFB equations $(17|19| 20|26| 27 \mid 28)$ - note that this is not consistent with Eq. (21) and leads to the violation of conservation laws. This solution has been popularised as the HFB-Popov approximation in the recent literature on magnetically trapped BoseEinstein condensates [17].

However, the importance of the anomalous average near the ground state has been stressed by some authors [18, 19, 20] who devised new variations of the HFB approximation in order to include that average.

In the "improved HFB" approach [18, 19] it is argued that 

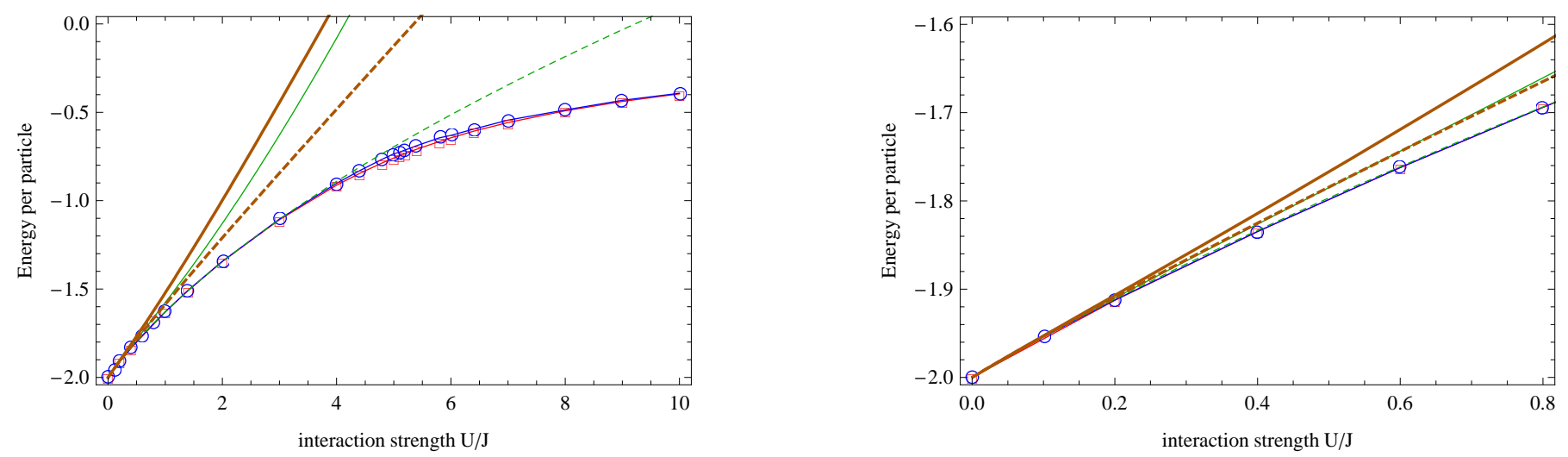

Figure 5: Energy for $N=60, M=60$ as a function of $U / J$. The right panel is a close-up view of the weakly-interacting regime. The same conventions as those of Fig. 2 are used.
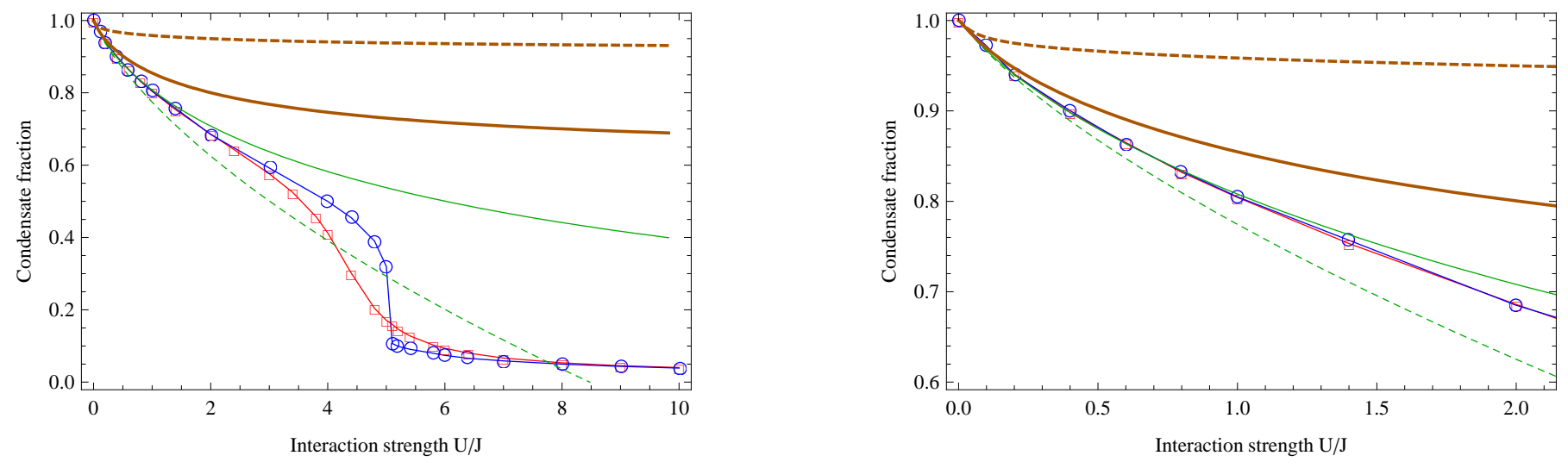

Figure 6: Condensate fraction for $N=60, M=60$ as a function of $U / J$. The right panel is a close-up view of the weakly-interacting regime. The same conventions as those of Fig. 2 are used.

the decoupling of noncondensate particles is too strong an assumption, resulting in noncondensate particles interacting through a bare $T$-matrix. On the other hand, the condensate particles interact through a full $T$-matrix, renormalised by the presence of $\tilde{m}$, which is a measure of condensate-condensate correlations. If one assumes that the noncondensate correlations neglected in the HFB approximation amount to something similar to $\tilde{m}$, it seems reasonable that the bare $T$-matrix for noncondensate particles should be upgraded to the same renormalised $T$-matrix for condensate particles. This can be done by hand in the HFB equations by changing the sign in front of $\tilde{m}$ in Eq. (26). This way, the gap disappears.

In the representative statistical ensemble approach [20, 21], it is argued that the number of condensate particles $N_{0}$ deserves a special treatment because it appears as a new macroscopic quantity in the system. As a result, a new chemical potential $\mu_{0}$ associated to $N_{0}$ should be introduced. It is distinct from the chemical potential $\tilde{\mu}$ associated to the number of nonconden-

\section{THE VARIATIONAL BIJL-DINGLE-JASTROW METHOD}

We now consider a variational method. It consists in minimizing the energy of the system within a subspace of the full sate particles $\tilde{N}=N-N_{0}$. This distinction changes Eq. 26. to

$$
\omega_{q}=\left(n_{0}-\tilde{m}\right) U+\left(\mu_{0}-\tilde{\mu}\right)+4 J \sin \left(\frac{\pi q}{M}\right)^{2} .
$$

If one chooses $\mu_{0}-\tilde{\mu}=2 \tilde{m} U$, the HFB equations become gapless. Note that this results in the same modification as in the "improved HFB" approach. Although the two approaches are conceptually different and do lead to different results in more general cases (such as nonlocal interactions), they yield the same equations in the case studied in this paper.

In both cases, the energy is obtained by formally changing the sign of $\tilde{m}$ :

$$
E_{H F B Y}=E_{B o g}-U \tilde{n} \tilde{m}+\frac{1}{2} U\left(\tilde{n}^{2}-\tilde{m}^{2}\right) .
$$

Hilbert space. The minimization is numerically possible if that subspace is not too large, and leads to a good approximation of the true ground state if it is general enough to allow the important features of that state. As recently proposed in Ref. [24], we 

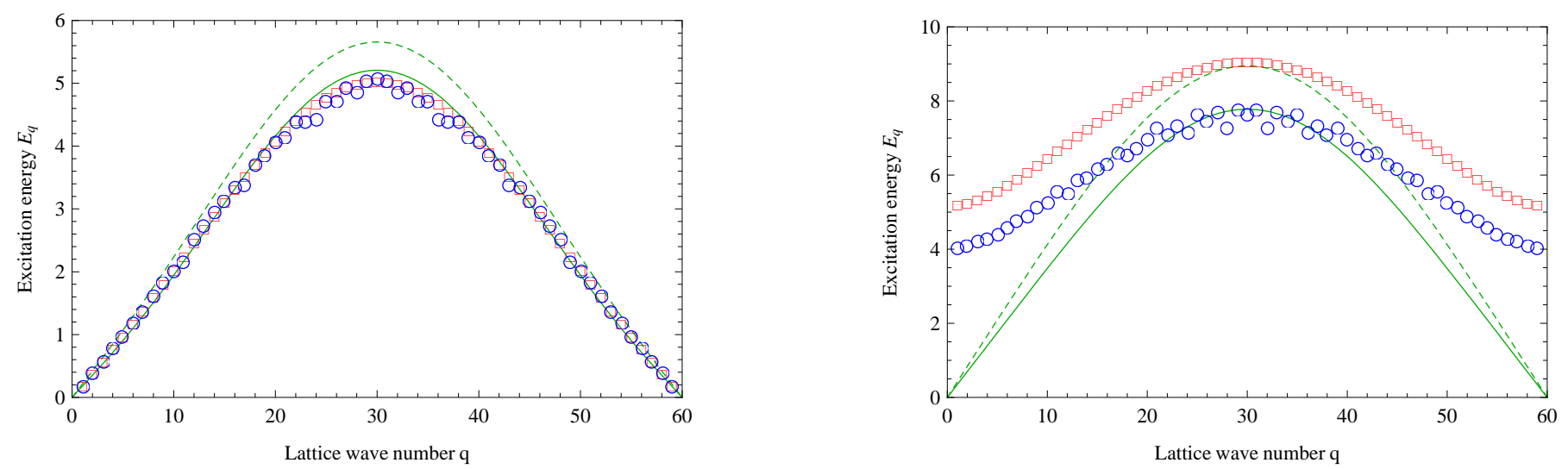

Figure 7: Excitation spectrum for $N=60, M=60$. The same conventions as those of Fig. 2 are used. The left panel corresponds to $U / J=2$ and the right panel corresponds to $U / J=8$. Note that the apparent dispersion of the blue circles are residual fluctuations due to the stochastic nature of the variational Monte-Carlo method.

choose the subspace of wave functions having the Bijl-DingleJastrow form [22, 23]:

$$
\Psi\left(x_{1} \ldots x_{N}\right)=\prod_{i<j} f\left(x_{i}-x_{j}\right)
$$

This is a simple form obtained by a product of some correlation function $f$ for all pairs of bosons. The Bijl-Dingle-Jastrow form Eq. 31 is more general than the structure of the Bogoliubov ground state (which can be reproduced from Eq. (31) in the limit of weak interaction). Thus it can be applied successfully to more strongly-interacting regimes. However, this method gives only the ground-state properties and some excitation properties, unlike the previous methods which are also applicable at finite temperature. Another drawback is that the minimum cannot be found analytically.

To find the minimum numerically, we use the methods described in Ref. [24]. Namely, minimization is performed by means of the power method, and at each step a Monte-Carlo algorithm computes all the average quantities relevant to the minimization procedure. Once the optimal $f$ is found, any observable can be obtained by taking the average of the corresponding operator in the quantum state $\Psi$. This average is calculated using the Monte-Carlo algorithm.

\section{RESULTS}

We apply the methods described in the previous sections to solve the Bose-Hubbard model in two cases: an incommensurate case $N=48, M=60$ (for which the lattice is filled to $80 \%$ ) and a commensurate case $N=60, M=60$ (for which the lattice is filled to $100 \%$ ). The Superfluid-Mott insulator transition occurs only in the commensurate case. In both cases, we look at characteristic properties of the ground state such as its energy per particle $E$ and its condensate fraction $n_{0}$, as well as the excitation spectrum $E_{q}$. In the extended Bogoliubov methods, these quantities appear naturally as variables of the problem - see Eqs. (28), (19) and (25). In the TEBD and Bijl-Dingle-Jastrow methods, the energy is obtained by calculating the average $\langle\hat{H}\rangle$, and the condensate fraction is given by $N_{0} / N$, where the number of the condensate bosons $N_{0}$ is obtained from the largest eigenvalue of the one-body density matrix $\left\langle\hat{a}_{j}^{\dagger} \hat{a}_{l}\right\rangle$ [29]. A very close upper bound of the excitation spectrum $E_{k}$ is obtained from the $f$-sum rule [40], which states that

$$
E_{q} \lesssim K \frac{-2 \sin \left(\frac{\pi q}{M}\right)^{2}}{S_{q}}
$$

where both the kinetic energy $K=\left\langle-J \sum_{i=1}^{M}\left(\hat{a}_{i}^{\dagger} \hat{a}_{i+1}+\hat{a}_{i+1}^{\dagger} \hat{a}_{i}\right)\right\rangle$ and the structure factor $S_{q}=\frac{1}{N} \sum_{i, j}\left(\left\langle\hat{n}_{i} \hat{n}_{j}\right\rangle-\left\langle\hat{n}_{i}\right\rangle\left\langle\hat{n}_{j}\right\rangle\right) e^{i q(i-j)}$ can be calculated by quantum averages.

Results for the incommensurate case are shown in Figs. 2, 3 and 4 One can see that there is excellent agreement between the Bijl-Dingle-Jastrow calculations and the TEBD results for any interaction strength. On the other hand, all the Bogoliubov methods agree with these results only in the weakly-interacting limit $U / J \lesssim 0.2$. Curiously enough, the energy in the Bogoliubov approximation shows very good agreement with the TEBD and Bijl-Dingle-Jastrow energy, even for strong interactions $U / J \gg 0.2$. While this might provide some analytical insights to devise a more elaborate theory, we believe that this is most likely a mere coincidence, since the Bogoliubov condensate fraction is correct only for $U / J \lesssim 0.2$. The same applies for the Popov approximation which reproduces the correct condensate fraction up to $U / J \lesssim 2$ but fails to predict the average energy for $U / J \gtrsim 0.2$.

Results for the commensurate case are shown in Figs. 5 . 6 and 7 One can see that a quantum phase transition for the superfluid phase to the Mott-insulator phase occurs around $U / J=4$. Since we are dealing with a finite-size system, the transition is smoothed. All the extended Bogoliubov methods fail to reproduce that transition, since their range of validity is limited to $U / J \lesssim 0.2$, but again, the Bogoliubov approximation shows a surprisingly excellent prediction of the energy over the whole superfluid regime. As it was found in Ref. [24], the BijlDingle-Jastrow method does show a quantum phase transition and accurately predicts the energy and condensate fraction in the strongly-interacting limit of the Mott-insulator regime. The excitation spectrum in Fig. 7 also shows that a gap appears in the Bijl-Dingle-Jastrow method, while all the extended Bogoliubov spectra remain gapless (by construction).

While these results seem to indicate that the Bijl-Dingle- 


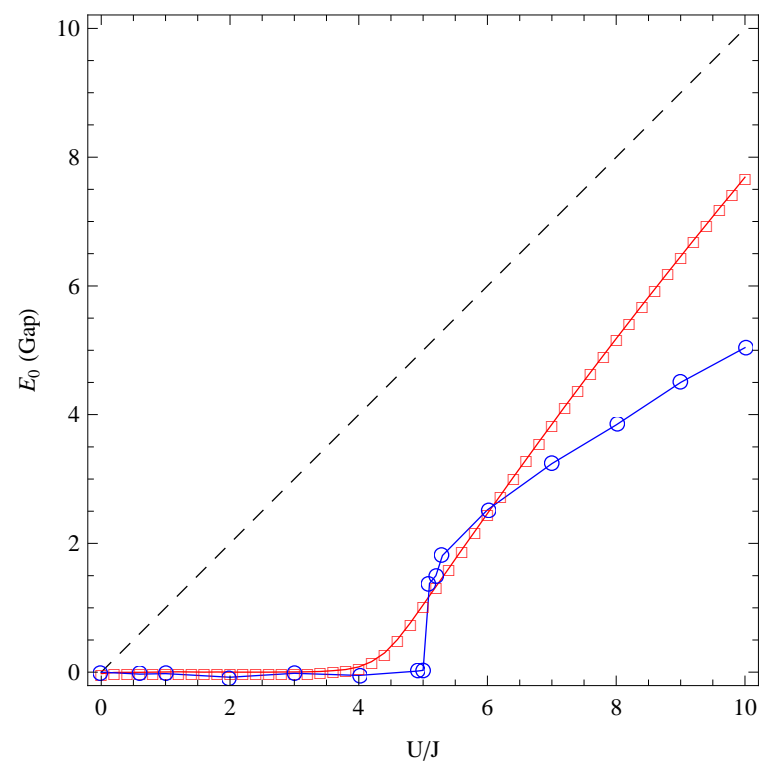

Figure 8: Gap in the excitation spectrum ( $E_{q}$ for $q=0$ ) as a function of $U / J$, for $N=60, M=60$. The red squares represent the TEBD results, and the blue circles correspond to the variational Bijl-DingleJastrow method.

Jastrow ansatz is able to reproduce the superfluid-to-Mott insulator transition, as claimed in [24], it should be noted that it does only in a superficial way. Firstly, the transition itself is only approximately reproduced by the Bijl-Dingle-Jastrow method, which predicts a somewhat surprisingly sharper transition than the actual one and at a different value of $U / J$ around 5. This fact is expected since the system is known to be strongly correlated near the transition, in a way which is hardly recovered by any simple ansatz. Secondly, although the BijlDingle-Jastrow ansatz tends towards the exact noninteracting ground state when $U / J \rightarrow 0$, it does not tend towards the exact Mott insulator state when $U / J \rightarrow \infty$. Indeed, while it can repro- duce accurately the energy and condensate fraction, it fails to reproduce the excitation gap, which even departs further from the real one as $U / J \rightarrow \infty$, as shown in Fig. 8 . From this we conclude that the Bijl-Dingle-Jastrow ansatz provides a partial description a Mott-insulator, the usefulness of which is restricted to the calculation of quantities deriving from low-order correlations.

\section{CONCLUSION}

We have performed an extensive comparison of several theoretical treatments of the Bose-Hubbard Hamiltonian ground state, describing bosonic atoms interacting in an optical lattice. The TEBD method was generalised to periodic boundary conditions in order to solve the problem. It was used as a quasiexact reference to compare with the results of extended Bogoliubov methods and the variational Bijl-Dingle-Jastrow ansatz.

We showed that all the methods refining the original Bogoliubov approximation do not in fact bring any significant improvement. The validity of all these methods is restricted to the weakly-interacting regime $U / J \lesssim 0.2$. On the other hand, the Bijl-Dingle-Jastrow ansatz proves to be a very accurate approximation of the ground state in the superfluid phase for any strength of the interaction, showing that particles in such a phase are essentially correlated by pair. However, it gives only a partial account of the ground state in the Mott insulator phase, showing that more-than-two-particle correlations are needed in that phase. While the structure of the Mott insulator in the strongly-interacting regime is known, devising a theory which can account for both the superfluid and Mott insulator phases accurately is still a challenging problem.

\section{Acknowledgments}

I. D. is supported by a Grant-in-Aid from JSPS.
[1] I. Bloch, Nat. Phys. 1, 23 (2005).

[2] O. Morsch and M. Oberthaler, Rev. Mod. Phys. 78, 179 (2006).

[3] M. Lewenstein, A. Sanpera, V. Ahufinger, B. Damski, A. Sen, and U. Sen, Adv. Phys. 56, 243 (2007).

[4] M. P. A. Fisher, P. B. Weichman, G. Grinstein, and D. S. Fisher, Phys. Rev. B 40, 546 (1989).

[5] D. Jaksch et al., Phys. Rev. Lett. 81, 3108 (1998).

[6] M. Greiner, O. Mandel, T. Esslinger, T. W. Hänsch, and I. Bloch, Nature (London) 415, 39 (2002).

[7] T. Stöferle, H. Morzitz, C. Schori, M. Köhl, and T. Esslinger, Phys. Rev. Lett. 92, 130403 (2004).

[8] I. B. Spielman, W. D. Phillips, and J. V. Porto, Phys. Rev. Lett. 98, 080404 (2007).

[9] L. De Sarlo, L. Fallani, J. E. Lye, M. Modugno, R. Saers, C. Fort, and M. Inguscio, Phys. Rev. A 72, 013603 (2005).

[10] J. Mun, P. Medley, G. K. Campbell, L. G. Marcassa, D. E. Pritchard, and W. Ketterle, Phys. Rev. Lett. 99, 150604 (2007).

[11] K. Xu, Y. Liu, D. E. Miller, J. K. Chin, W. Setiawan, and W. Ketterle, Phys. Rev. Lett. 96, 180405 (2006).

[12] I. B. Spielman, W. D. Phillips, and J. V. Porto, Phys. Rev. Lett.
100, 120402 (2008).

[13] N. N. Bogoliubov, J. Phys. (USSR), 11(23) 1927. Reprinted in The many-body problem, edited by D. Pines (W. A. Benjamin, New York, 1961).

[14] D. A. Huse and E. D. Siggia, J. Low Temp. Phys., 46, 137 (1982).

[38] M. Girardeau and R. Arnowitt, Phys. Rev. 113, 755 (1959).

[16] N. Shohno, Progr. Theor. Phys. 31553 (1964).

[17] A. Griffin, Phys. Rev. B, 5314 (1996).

[18] N. P. Proukakis, S. A. Morgan, S. Choi, and K. Burnett, Phys. Rev. A 58, 2435 (1998).

[19] D. A. Hutchinson, K. Burnett, R. J. Dodd, S. A. Morgan, M. Rusch, E. Zaremba, N. P. Proukakis, M. Edwards, and C. W. Clark, J. Phys. B 33, 3825 (2000).

[20] V. I. Yukalov and E. P. Yukalova, Phys. Rev. A 74, 063623 (2006).

[21] V. I. Yukalov, Ann. Phys. 323, 461 (2008).

[22] A. Bijl, Physica 7, 869 (1940)

[23] R. Jastrow, Phys. Rev. 98, 1479 (1955).

[24] M. Capello, F. Becca, M. Fabrizio, and S. Sorella, Phys. Rev. 
A 99, 056402 (2007); M. J. Capello, Ph.D. thesis, Scuola Internazionale Superiore di Studi Avanzanti Trieste, 2006.

[25] G. Vidal, Phys. Rev. Lett. 91, 147902 (2003); ibid. 93, 040502 (2004).

[26] S. R. White, Phys. Rev. Lett. 69, 2863 (1992); Phys. Rev. B 48, 10345 (1993).

[27] U. Schollwöck, Rev. Mod. Phys. 77, 259 (2005).

[28] G. Vidal, Phys. Rev. Lett. 98, 070201 (2007).

[29] O. Penrose and L. Onsager, Phys. Rev. 104, 576 (1956).

[30] Y.-Y. Shi, L.-M. Duan, and G. Vidal, Phys. Rev. A 74, 022320 (2006).

[31] I. Danshita, J. E. Williams, C. A. R. Sá de Melo, and C. W. Clark, Phys. Rev. A 76, 043606 (2007); I. Danshita, C. A. R. Sá de Melo, and C. W. Clark, Phys. Rev. A 77, 063609 (2008).

[32] L. Mathey, I. Danshita, and C. W. Clark, arXiv:0806.0461 (2008).

[33] A. J. Daley, S. R. Clark, D. Jaksch, and P. Zoller, Phys. Rev. A 72, 043618 (2005); A. J. Daley, Ph.D. thesis, Leopold-FranzensUniversität Innsbruck, 2005 (unpublished).
[34] D. van Oosten, P. van der Straten, and H. T. C. Stoof, Phys. Rev. A 63, 053601 (2001).

[35] L. P. Pitaevskii and S. Stringari, Bose-Einstein Condensation (Oxford University Press, Oxford, 2003).

[36] T. Kinoshita, T. Wenger, and D. S. Weiss, Science 305, 1125 (2004).

[37] E. H. Lieb and W. Liniger, Phys. Rev. 130, 1605 (1963); E. H. Lieb, Phys. Rev. 130, 1616 (1963).

[38] M. Girardeau, J. Math. Phys. 1, 516 (1960).

[39] A. L. Fetter and J. D. Walecka, Quantum Theory of Many Particle Systems, McGraw-Hill, Boston (1971).

[40] G. G. Batrouni, F. F. Assaad, R. T. Scaletter, and P. J. H. Denteneer, Phys. Rev. A 72, 031601(R) (2005).

[41] These durations are those which were necessary for the calculations presented in this paper and are mentioned to give some idea to the reader, although it should be noted that they strongly depend on the computional environment and required accuracy. 